\title{
ADVANTAGES OF PRE-PROSTHETIC MASTICA- TORY SYSTEM PREPARATION WITH OCCLUSAL APPLIANCE
}

\author{
Mariana Yankova ${ }^{1}$, Desislava Dimitrova ${ }^{2}$, Todor Peev ${ }^{3}$, Bozhidar Yordanov ${ }^{1}$, \\ 1) Department of Prosthetic Dentistry, Faculty of Dental Medicine, Medical \\ University - Sofia, Bulgaria. \\ 2) Dental Clinic “Edinstvo"-Sofia, Bulgaria. \\ 3) Private Practice, Sofia, Bulgaria.
}

\begin{abstract}
:
The aim of this literature review is to present the main advantages of pre-prosthetic preparation of the masticatory system with occlusal appliances.

The possibilities for attaining muscle relaxation at the achieved centric condyle position in the temporomandibular joints are discussed. The advantages, in terms of hard dental tissue protection, the diagnostic value and the psychoprophylactic effect, are considered.

The wide-ranging benefits of the use of occlusal appliances in bruxism- and bruxomania-related craniomandibular dysfunctions are indisputable and represent a current scientific interest.
\end{abstract}

Keywords: occlusal appliances, craniomandibular dysfunction, advantages,

\section{INTRODUCTION:}

Pre-prosthetic preparation of the masticatory system in patients with craniomandibular dysfunctions, performed after the recording of patients' individual values, is a subject of scientific interest $[1,2]$. Temporomandibular joints (TMJs) and their three-dimensional positioning in articular fossae are an important factor for normal function and have an impact on body posture [3,4]. Permanent spatial deviations in TMJs are known to cause strain on structures, such as the bilaminar zone, the articular disc, ligaments and bone structures. The result is a neuromuscular protective reaction within the masticatory system and the buffer zones of the adjacent organ systems (abnormal position of the head, spine, pelvis, etc.) $[5,6]$.

It is known that the physiological position of the TMJs is maintained by the static occlusion. In the case of impaired occlusal-articulation relationships, there is a need to create new occlusal conditions by using appliances, i.e. splints [7], which allow redistribution of occlusal forces and protect the muscles from hyperactivity $[8,9]$.

The aim of this literature review is to present the main advantages of pre-prosthetic preparation of the masticatory system with occlusal appliances.

\section{RESULTS AND DISCUSSION:}

The Glossary of Prosthodontic Terms [10] defines the occlusal appliance (splint) as a removable device with occlusal surfaces intended for diagnosing or treating mandible-to-maxilla relationships. A properly constructed occlusal appliance maintains the harmony in the relationships between the masticatory muscles, the articular disc, the joints, the ligaments, the bone structures, the teeth and the tendons. It provides easy and non-invasive prerequisites for reversible changes in occlusion. For example, Slavicek [11] believes that the myopathic occlusal appliance should release the existing occlusion by eliminating the relationships between the upper and lower teeth and the existing prematurities. As a result, reference positions in the TMJs and muscle relaxation are achieved. The decompressing myopathic occlusal appliance fulfills this role and additionally allows the unloading of the TMJs. In patients with loss of occlusion height due to bruxism, the author recommends the vertical occlusal appliance. He also describes the repositioning occlusal appliance in cases of changes in the relationships and the starting position in the TMJ.

There are known various classifications of occlusal appliances $[11,12,13]$. The classification criteria are different: action and duration of use of the appliance, materials for fabrication, choice of the jaw for adjusting the appliance, etc.

Occlusal appliances are the most common initial, a non-invasive therapeutic approach used to treat patients with craniomandibular dysfunctions [14]. They protect the teeth from damage. Their use leads to the reduction of masticatory muscle contraction and orofacial pain $[12,15]$.

Today, there is no explicit consensus on the clinical indications and the functioning of occlusal appliances. Their psychoprophylactic effect, possible placebo effect, changes in masticatory muscle function resulting from changes in the occlusion and mandibular movement, the effect of determining the centric condyle position, and others are discussed [16, 17]. A number of authors $[18,19,20]$ define occlusal appliances as a conservative, non-invasive approach in the treatment of patients with craniomandibular dysfunctions. 
Occlusal appliances allow for muscle relaxation and repositioning in the joint. This defines them as a means of promoting the diagnostic effect. Their effect is considered to be completely reversible, and the treatment approach with occlusal appliances can be considered as non-invasive.

All occlusal plates and appliances aim at a temporary deprogramming effect, which in turn causes a new adaptation to a stable occlusal position. If the splint positions correct relationships in the TMJs and builds up correct occlusal-articulation relationships, the results are satisfactory. Otherwise, it triggers the development of a new adaptation mechanism, and the patient's condition may rapidly deteriorate (causing increased muscle activity) or dramatically improve (decreasing the hypertonus by about $20 \%$ ) and then rapidly reverse.

It can be summarized that pre-prosthetic preparation with occlusal appliances is used for the diagnosis and treatment of many disorders of the craniomandibular system [21]. Occlusal appliances are administered to patients with bruxism and additional symptoms such as tension and pain in the facial, oral, head, neck and other regions. A common reason for the use of occlusal appliances is the protection of teeth from occlusal wear. Treatment with occlusal appliances aims at protecting the structures of the temporomandibular joint from the deleterious effects of excessive non-functional forces. Other treatment goals are to normalize the masticatory muscle function and to relieve the pain by creating a stable, balanced occlusion. According to Williamson [22], the hyperactivity of the muscle group of jaw-closures is reduced with the removal of distal extrusion contacts by creating frontal guidance.

An important advantage with the use of occlusal appliances is the achievement of a centric condyle position in both TMJs. The TMJs and their three-dimensional positioning relative to articular fossae are the key parameters for a trouble-free function, not only as part of the masticatory system but also as a receptor and a mediator of the whole body posture [3, 4]. However, it should be borne in mind that in patients with craniomandibular dysfunction (CMD), central occlusion (habitual occlusion) is usually pathological for the patient, as it performs parafunctions. The occlusal parameters are lost, and to restore the physiological norm relationships, it is necessary to register a reproducible, physiological position in the TMJs. This position is individually tailored and is characterized by the "ideal" three-dimensional position of the articular head-articular disc complex in the articular fossae of both TMJs.

From the beginning of the 20th century to this day, the understanding of central relation has evolved from a "strained most posterior position" through "unstrained most posterior position" and "most superior position" to the modern understanding of a "cranio-ventral" position. The idea of the articulation axis has also changed from a "terminal articulation axis" to a "centered articulation axis".

This general change in the knowledge that central relation is not a retrusive or retral position, either "strained" or "unstrained", is due to advances in instrumental graphical recording methods (EPA-test) and the development of non-invasive diagnostic imaging (MRI).

In Bulgaria, in 2015, Dimova [23] introduced the modern definition of centric condyle position and put it into practice. According to the author, "Centric condyle position is an individually tailored, reproducible relationship in both TMJs, where the articular head-articular disc complex is in the anterior-superior, laterally non-displaced position against the slopes of eminentia articularis, and the articular head is located in the intermediate zone of the disc under the conditions of a physiological tissue load and coordinated muscle tonus. Centric condyle position is a stable musculoskeletal position, from which the only movement of rotation is possible with the formation of single or multiple weak contacts between antagonizing teeth" [23].

The accurate registration of centric condyle position is of particular importance, especially in patients with craniomandibular dysfunction, as it is the therapeutic position of the mandible in which the masticatory system should be relaxed and pre-prosthetically prepared.

The centric condyle position is the most stable position for determining and performing dental row restorations. The centric registration allows for the fabrication of occlusal appliances that aim to relax the jaw-closing muscles. Such pre-prosthetic preparation favors the location of the articular head-articular disc complex in the anterior-superior position in the TMJ.

Modern medicine considers headache as an interdisciplinary problem and admits the existence of interactions between functional disorders of the masticatory system and nonspecific headaches, tension-related headaches and trigeminal neuralgia. Disregarding chronic, persistent problems and dysfunctions in the TMJs, masticatory muscles and muscles of the floor of the oral cavity is the most common reason for compromising the treatment of facial pain, headache and vertigo. These complaints significantly impair patients' quality of life, both mentally and socially. Statistically significant correlations between the presence of facial pain and the factors of headache, neck, chest and back pain have been demonstrated. According to Bumann and Lotzmann [24], signs and symptoms of CMD were diagnosed in $50 \%$ of patients with trigeminal neuralgia.

Evidence for the relationship between the primary headache, migraine, and myogenic craniomandibular dysfunction is provided by a large-scale study in Pomerania (SHIP) [25], involving over 4,000 subjects. The observed significant correlations lead to the conclusion that in CMD, masticatory muscles that are painful on palpation are a possible risk factor for $92 \%$ of headaches. These results are confirmed by numerous clinical and epidemiological studies. For example, the studies of Molina et al. [26], conducted in two hundred sixty-six patients (one hundred thirty-three people with myoarthropathies and one hundred thirty-three people without functional pathology of the masticatory system), have shown that headache is more common in patients with myoarthropathies $(76 \%)$ than in those without myoarthropathies (49\%). In both groups, headaches associated with a feeling of tension (36\% in the first and $21 \%$ in the second group, respectively) were prevailing over a migraine $(12 \%$ of patients in the first group and only $2 \%$ 
of patients in the second group).

There are studies that examine the relationship between headache and craniomandibular dysfunction in terms of subjective perception of pain and emphasize that intense headache can be felt by the patients as a pain in the masticatory muscles.

Muscle fatigue due to prolonged hypertonus in patients with craniomandibular dysfunctions is often manifested with pain. It is known [27] that headaches associated with impaired function of the craniomandibular system can be significantly reduced with the use of occlusal appliance therapy. For example, the nociceptive trigeminal inhibiting occlusal appliance is a means for preventing migraine headache [28]. Pre-prosthetic preparation with an occlusal appliance aims at even distribution of dental contacts and relaxation of the muscles. Today, it is considered [11,22] that providing disocclusion in the distal sections of the dentition during the excursive movements of the mandible - protrusion and laterotrusion - leads to muscle relaxation, reduction and disappearance of pain symptoms $[12,28]$.

Electromyographic studies have conclusively confirmed muscle relaxation and have shown that, when eliminating occlusal interferences, muscle hyperactivity is reduced.

Another advantage of pre-prosthetic preparation with occlusal appliances is the protection of hard dental tissues from the effects of bruxism. According to Georgiev [29], severe untreated tooth wear leads to the appearance of symptoms of functional pathology in the TMJs. Dimova [23] conducted a survey on the use and effectiveness of occlusal appliances used in bruxism. The results have shown a low relative proportion of the dental practitioners treating bruxism and bruxomania $(17.84 \%-23.87 \%)$. Most often, the treatment was performed by using occlusal appliances (splints). It is noteworthy that $60.5 \%$ of the respondents believe that the treatment provided does not improve the patient's condition [30,31].

Modern studies [32,33] determine the magnitude of forces in bruxism. Bruxism has been found to alter the maximum bite strength. The factor relevant to this change is the presence of pain symptoms in the structures of the masticatory system.

Occlusal appliances, administered to patients with bruxism, do not prevent its manifestation but distribute the forces generated by it evenly [24]. The purpose of the occlusal appliance is to redistribute these forces to the dentition and redirect them to the other structures of the masticatory system. Some studies $[15,34]$ have shown a reduction in the incidence of bruxism episodes when using occlusal appliances.

On the other hand, the use of occlusal appliances allows for avoiding harmful prematurities and imbalances. Some authors [35] define occlusion as a fine system with neurological feedback that directs the movements of the mandible in relation to the teeth of the maxilla. At the same time, occlusal contacts maintain the position of the condyle in a physiological position $[5,6]$. Today, it is known that occlusal sensitivity ranges from $0.02-0.03 \mathrm{~mm}$
$[35,36]$. On the other hand, the reported tolerance of deviations in the TMJ is only $0.6-0.8 \mathrm{~mm}[5,6]$. However, these are average values, and the dental practitioner should always take into account the individual characteristics of each clinical case. All these increase the requirements to the dental practitioner in the complex and ambiguous field of functional diagnostics of the masticatory system. The choice of a non-invasive initial therapy, performed with occlusal appliances, with the possibility of complete reversibility, is mandatory in patients with occlusion-preconditioned craniomandibular dysfunction [14, 37]. When patients seek consultation and treatment with a previously developed pathological clinical picture, and the presence of severe pain symptoms, the initiation of invasive treatment is contraindicated. The first step and challenge for the dental practitioner is to achieve relaxation of the masticatory system. This means that measures should be taken to significantly reduce subjective symptoms in order to accurately record the centric condyle position. When the preprosthetic preparation with occlusal appliances results in the objectively detectable reduction of the clinical signs and the subjective assessment of the patient alleges a lack or a significant reduction of the pain symptoms, the definitive restoration of correct occlusal-articulation relationships at a certain occlusion height and central position of the mandible may proceed $[1,11,36,38]$.

Another advantage with the use of occlusal appliances is the possibility to provide diagnostic information about the pattern of occlusal wear, which depends on the chewing habits. The study of Holmgrenetal [34], determining the pattern of occlusal wear in patients with bruxism, has shown teeth clenching in 13\%, unilateral displacement in $71 \%$ and predominant protrusive movements in $3 \%$ of the subjects. Occlusal appliances made of hard resin were used to conduct the study. Patterns of occlusal wear, observed on occlusal appliances, provide information that may be considered when composing a treatment plan for the final prosthetic restoration. The neutral zone, the axial axes of loading, the occlusal configuration, the shape and size of tubercles and the angulation of guidance are analyzed. The choice of suitable prosthetic materials is made.

The use of occlusal appliances is believed to decrease the proprioceptivity of the periodontal ligament, and this is also considered an advantage of their application. It is known that the root surface of each tooth is covered with the periodontal ligament and, upon stimulation by the masticatory forces, a nerve impulse is sent to the central nervous system [39]. As a result, information is sent via efferent pathways to the masticatory muscles, and the tooth structures are prevented from overloading. In this process, the role of the occlusal appliance is to redistribute the masticatory forces by covering all the occlusal surfaces and cutting edges. It relies on the uniform force distribution system of the teeth and the effect of the total periodontium. Regular adjustment of occlusal appliances is recommended in order to achieve a uniform distribution of the contacts and to maintain the balance.

The use of occlusal appliances reduces the levels of 
hypoxia in the cells. The difference in capillary perfusion with and without the use of an occlusal appliance was determined [40], and it was found that without an occlusal appliance, the capillary pressure exceeded $200 \mathrm{mmHg}$, while the pressure reported with an occlusal appliance was significantly lower $(20 \mathrm{mmHg})$.

The psychoprophylactic effect with the use of occlusal appliances is also important. Reducing pain symptoms increases the patient's confidence in the treatment and enhances his or her motivation to continue the treatment according to the approved treatment plan.

\section{CONCLUSION:}

Due to its complexity, functional disorders of the masticatory system are a difficult area in the diagnostic and treatment plan. The wide-ranging benefits of the use of occlusal appliances in bruxism- and bruxomania-related craniomandibular dysfunctions are indisputable and of current scientific interest.

\section{REFERENCES:}

1. Dimova M. Occlusion and articulation in bruxism and bruxomania investigated with the system T-scan. $J$ of IMAB. 2014 Oct-Dec;20(5):655-660. [Crossref]

2. Murali R, Rangarajan $P$, Mounissamy A. Bruxism: Conceptual discussion and review. J Pharm Bioallied Sci. 2015 Apr;7(Suppl 1): S265-70. [PubMed]

3. Lee Y-J, Park J-H, Lee S-J, Ryu H-M, Kim S-k, Lee Y-J, et al. Systematic Review of the Correlation Between Temporomandibular Disorder and Body Posture. J Acupunct Res. 2017; 34(4): 159-168. [Crossref]

4. Costen JB. A syndrome of ear and sinus symptoms dependent upon disturbed function of the temporomandibular joint. Ann Otol Rhinol Laryngol. 1997 Oct;106(10 Pt 1):80519. [PubMed]

5. Christiansen G. Computer-aided measurement of the functional joint space of the temporomandibular joint. J Craniomand Func. 2014 Winter; 2(4):329-43.

6. Greven M, Otsuka T, Zutz L, Weber B, Elger C, Sato S. The amount of TMJ displacement correlates with brain activity. Cranio. 2011 Oct;29(4):2916. [PubMed]

7. Zhang C, Wu JY, Deng DL, He BY, Tao Y, Niu YM, et al. Efficacy of splint therapy for the management of temporomandibular disorders: a metaanalysis. Oncotarget. 2016 Dec 20; 7(51):84043-84053. [PubMed]

8. Yap AU, Chua AP. Sleep bruxism: Current knowledge and contemporary management. J Conserv Dent. 2016 Sep-Oct;19(5):383-389. [PubMed]

9. Racich MJ. Occlusion, temporomandibular disorders, and orofacial pain: An evidence-based overview and update with recommendations. $J$ Prosthet Dent. 2018 Nov;120(5):678685. [PubMed]

10. Morgano SM, VanBlarcom CW, Ferro KJ, Bartlett DW. The history of the glossary of Prosthodontic terms. $J$ Prosthet Dent. 2018 Mar;119(3):311312. [PubMed]

11. Dimova-Gabrovska M. Algorithm for Computerized Analysis of Static, Dynamic and Functional Occlusion in Patients with Bruxism and Bruxomania. C R Acad Bulg Sci. 2019; 72(2):259-266. [Crossref]

12. Okeson JP. Management of temporomandibular disorders and occlusion. 5th ed. St. Louis:Mosby; 2003. 97 p.

13. Dawson PE. Evaluation, diagnosis and treatment of occlusal problems. 2nd Edition. St. Louis;Mosby, 1989. 186 p.

14. de Kanter RJAM, Battistuzzi PGFCM, Truin GJ. Temporomandibular Disorders: "Occlusion" Matters! Pain Res Manag. 2018 May 15;2018: 8746858. [PubMed]

15. Mehta NR, Forgione AG, Maloney G, Greene R. Different effects of nocturnal parafunction on the masticatory system: the weak link theory. Cranio. 2000 Oct;18(4):280-6. [PubMed]

16. Profoziæ A, Plazibat A, Polašek A, Pliško M, Èimiæ S. Position of Mandibular Condyles during Stabilization Splint Wearing. Acta Clin Croat. 2017 Dec;56(4):594-599. [PubMed]

17. Nilner M. Does splint therapy work for temporomandibular pain? Evid Based Dent. 2004; 5(3):65-6. [PubMed]

18. Glaros AG, Owais Z, Lausten L. Reduction in parafunctional activity: a potential mechanism for the effectiveness of splint therapy. J Oral Rehabil. 2007 Feb;34(2):97-104. [PubMed]

19. Amin A, Meshramkar R, Lekha K.Comparative evaluation of clinical performance of different kind of occlusal splint in management of myofascial pain. J Indian Prosthodont Soc. 2016 Apr-Jun;16(2):176-81. [PubMed]

20. Hasegawa Y, Kakimoto N, Tomita S, Fujiwara M, Ishikura R, Kishimoto $\mathrm{H}$, et al. Clinical study of splint therapeutic efficacy for the relief of temporomandibular joint discomfort. J Craniomaxillofac Surg. 2017 Nov; 45(11):1772-1777. [PubMed]

21. Kreiner M, Betancor E, Clark GT. Occlusal stabilization appliance. Evidence of their efficacy. $J$ Am Dent Assoc. 2001 Jun;132(6):770-7. [PubMed]

22. Williamson EH, Lundquist DO. Anterior guidance: its effect on electromyographic activity of the temporal and masseter muscles. $J$ Prosthet Dent. 1983 Jun;49(6):816-23. [PubMed]

23. Dimova-Gabrovska M. [Contemporary tendencies and gnathological preconditions in diagnosis and rehabilitation of craniomandibular disorders] [Dissertation] Medical University - Sofia. 2015. pp.50-217. [in Bulgarian].

24. Bumann A, Lotzmann U. TMJ Disorders and Orofacial Pain. Thieme Verlag; Stuttgart, 2002. 23 p.

25. Bernhardt O, Gesch D, Schwahn C, Mack F, Meyer G, John U, et al. Risk factors for headache, including TMD signs and symptoms, and their impact on quality of life. Results of the Study of Health in Pomerania (SHIP). 
Quintessence Int. 2005 Jan;36(1):5564. [PubMed]

26. Molina OF, dos Santos J Jr, Nelson SJ, Grossman E. Prevalence of modalities of headaches and bruxism among patients with craniomandibular disorder. Cranio. 1997 Oct;15(4):31425. [PubMed]

27. Manns A, Rocabado M, Cadenasso P, Miralles R, Cumsille MA. The immediate effect of the variation of anteroposterior laterotrusive contact on the elevator EMG activity. Cranio. 1993; 11(3):184-91. [PubMed]

28. Ghurye S, McMillan R. Orofacial pain - an update on diagnosis and management. Br Dent J. 2017 Dec;223(9):639-647. [PubMed]

29. Georgiev T. [Diagnosis and treatment of occlusal dental abrasion] [in Bulgarian]. Sofia. 1993: 103.

30. Dimova M, Arnautska H, Konstantinova D, Gerdzhikov I, Georgiev T, Yovchev D. Correlations between findings of occlusal and manual analysis in TMD-patients. J of IMAB. 2016 Jul-Sep;22(3):1242-1247. [Crossref]

31. Valcheva Z, Arnautska H, Dimova M, Ivanova G, Atanasova I.
The role of mouth breathing on dentition development and formation. $J$ of IMAB. 2018 Jan-Mar;24(1):1878-1882 [Crossref]

32. Dimova-Gabrovska M. [Bruxism. Functional characteristics of the masticatory system]. Varna: Medical University - Varna. 2017. 71 p. [in Bulgarian]

33. Palinkas M, Bataglion C, de Luca Canto G, Machado Camolezi N, Theodoro G, Siéssere $\mathrm{S}$ et al. Impact of sleep bruxism on masseter and temporalis muscles and bite force. Cranio. 2016 Sep;34(5):309-15. [PubMed]

34. Holmgren K, Sheikholeslam A, Riise C. Effect of a full-arch maxillary occlusal splint on parafunctional activity during sleep in patients with nocturnal bruxism and signs and symptoms of craniomandibular disorders. $J$ Prosthet Dent. 1993 Mar;69(3):293-7. [PubMed]

35. Shimazaki T, Otsuka T, Akimoto S, Kubo KY, Sato S, Sasaguri $\mathrm{K}$. Comparison of brain activation via tooth stimulation. J Dent Res. 2012 Aug;91(8):759-63. [PubMed]
36. Dimova M. Registration of centric occlusion in patients with bruxism and bruxomania through articulating paper and the system T-scan - comparative analysis. J of IMAB. 2014 JanJun;20(1):520-525. [Crossref]

37. Le Bell Y. Are occlusal treatments still possible and appropriate methods in clinical dentistry? $J$ Craniomand Func. 2014;6(4):317-32.

38. Robertson LT, Levy JH, Petrisor D, Lilly DJ, Dong WK. Vibration perception thresholds of human maxillary and mandibular central incisors. Arch Oral Biol. 2003 Apr;48(4):309-16. [PubMed]

39. Hannam AG, Wood WW, De Cou RE, Scott JD. The effects of working-side occlusal interferences on muscle activity and associated jaw movements in man. Arch Oral Biol. 1981; 26(5):387-92. [PubMed]

40. Nitzan DW. Intraarticular pressure in the functioning human temporomandibular joint and its alteration by uniform elevation of the occlusal plane. J Oral Maxillofac Surg. 1994 Jul;52(7):671-9. [PubMed]

Please cite this article as: Yankova M, Dimitrova D, Peev T, Yordanov B. Advantages of pre-prosthetic masticatory system preparation with occlusal appliance. J of IMAB. 2021 Apr-Jun;27(2):3731-3735.

DOI: https://doi.org/10.5272/jimab.2021272.3731

Address for correspondence:

Dr Mariana Yankova, PhD

Department of Prosthetic Dental Medicine, Faculty of Dental Medicine, Medical University - Sofia,

1, St. G. Sofiiski Blvd., Sofia, Bulgaria

e-mail: marianayankova13@gmail.com 